\title{
Dr. Guillermo E. Lema Fuxman Al Maestro, con cariño...
}

\author{
Roberto Canessa Be
}

Llegando el que había recibido cinco talentos, presentó otros cinco, diciendo: Señor, cinco talentos me entregaste; aquí tienes otros cinco que he ganado. Su señor le dijo: " iBien, siervo bueno y fiel!, en lo poco has sido fiel, al frente de lo mucho te pondré; entra en el gozo de tu señor" (Mateo 25, 20-21).

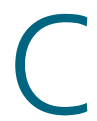
uando hace algunas semanas me comentaron que estaban planificando realizar este reconocimiento, pensé... "finalmente, llegó el momento...", no había que tener muchas dotes de adivino para sospechar que, más temprano o más tarde, esto tenía que ocurrir. Cuando a los pocos días me solicitaron hacer la "presentación bibliográfica" del homenajeado, me quedó claro que probablemente era una de las personas más indicadas para hacerlo y que no había ninguna posibilidad de eludir tamaña responsabilidad. Mal que mal, son más de 40 años de "vida en común", varios más que los que llevo de matrimonio.

Lo conocí, siendo yo un joven y esbelto interno de cirugía durante mi rotación por cirugía cardiotorácica, y él -residente de tercer año de Anestesia rotando por cardiovascular- armando y manejando una máquina llena de mangueras por donde circulaba sangre que pasaba de un color morado oscuro a un rojo rutilante después de literalmente burbujear en una especie de cafetera plástica, cosa que obviamente me causó gran asombro y que marcaría nuestras vidas para siempre. Al año siguiente, yo ya residente de Anestesia y él staff recién estrenado, lo tuve de modelo y profesor, para después de un tiempo terminar siendo compañeros de trabajo, de subespecialidad y de mil batallas de todo tipo, compartiendo la misma oficina por años (con todo lo que ello implica, muchas alegrías y también una que otra pena), fuimos vecinos de barrio, papás del mismo jardín infantil de los hijos, fue mi anestesiólogo en una sorpresiva cirugía con circulación extracorpórea hace unos años... y, finalmente, junto a mi señora, somos sus padrinos de bautizo, realizado obviamente cuando Guillermo ya era un adulto crecido y maduro... en fin, amigos de toda una vida. Por todo esto, agradezco enormemente al directorio de la Sociedad de Anestesiología de Chile el honor y el privilegio de haber sido designado para presentarlo en esta ocasión tan significativa.

Guillermo Eduardo Lema Fuxman, hijo de don Carlos Lema Mundaca, odontólogo de profesión, y de doña Clara Fuxman Kiguel, profesora de inglés (actualmente de 98 años), nace en la ciudad de Chillán el 19 de junio de 1954, en una familia que completaban su hermana mayor, Viviana, odontóloga como su padre y Fernando, el menor, ingeniero civil. Hizo sus estudios en el Liceo de Hombres de Chillán (actual Liceo Narciso Tondreau), donde Clarita, su madre, era profesora. Al final de la enseñanza media realiza una estadía formativa de 1 año en Portland, EE.UU., que como sabemos los más cercanos, sería muy importante en

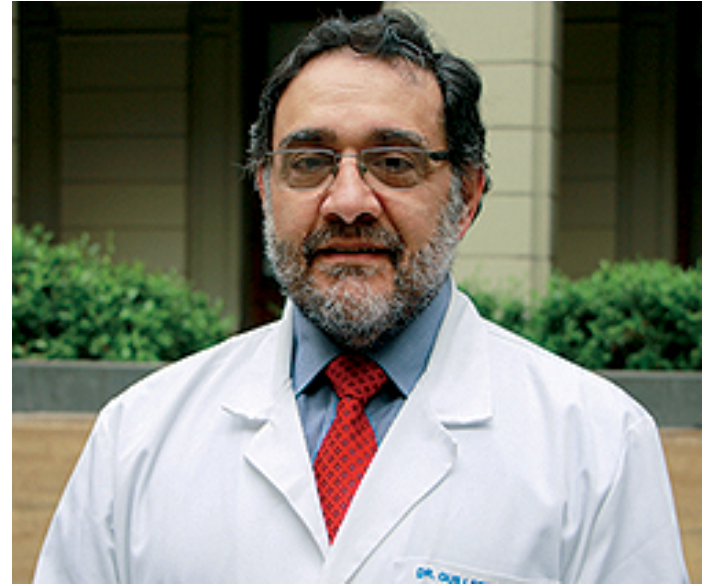

su vida actual, no solo por ayudarlo a optimizar su dominio del inglés. Terminado el colegio, se traslada a Santiago, para estudiar Medicina en la Universidad de Chile (sede Sur, Hospital Paula Jara Quemada o San Borja-Arriarán), entre los años 1972 y 1978. Bastaron esos pocos años para quedar impregnado de unos sentimientos un tanto incomprensibles por cierto equipito de fútbol (que aún no tiene ni estadio propio), sentimientos que lo acompañan hasta el día de hoy. Es frecuente encontrarlo en pasillos y oficinas entonando el "romántico viajero" en su celular. Como premonitorio de lo que sería su destacada carrera profesional y académica recibe, al egresar del pregrado, los premios "Colegio Médico" al mejor alumno de la carrera de Medicina de la promoción 1978 de la Universidad de Chile y "Fundación Aguilar" al mejor alumno de cirugía de su promoción.

Unos meses después comienza sus estudios de posgrado ingresando a la Beca de Anestesia de la Pontificia Universidad Católica de Chile, que cursaría entre los años 79 y 82, junto -entre otros- a su gran amigo y compadre Orlando Correa. En ese momento el Depto. de Anestesia de la UC estaba formado por Waldemar Badía (que partiría prontamente), Mario Allende, Viterbo González, Jorge Urzúa y los jóvenes José de la Fuente, Samuel Torregrosa, Jorge Dagnino y Ricardo Bustamante (este último que partiría luego también a la Posta Central). De estos 8 anestesiólogos, 6 han sido ya nombrados Maestros de la Anestesiología, lo cual habla del fértil ambiente académico que se vivía en aquellos años en la UC, muy propicio para el desarrollo de las buenas prácticas y la docencia de la especialidad.

Mientras estudiaba, Guillermo vivió en pensiones, contándose varias anécdotas de esa época, que por respeto a la familia (aquí presente) no entraremos a detallar. Finalmente, el año 1983 sienta cabeza y se casa con Janet Habash, tecnólogo médico, ma- 
trimonio del cual nacen sus 4 hijos: Nicolás, licenciado en Historia, con doctorados en Estética y Filosofía, actualmente trabajando en la Sorbonne, París, un "cabezón"; Tomás, psicólogo, viviendo en Australia, dedicado al turismo, un "gozador"; Constanza, la "niña de sus ojos", quien sigue sus pasos, recién recibida de médico en la Universidad Austral y comenzando una beca de Anestesia en la misma casa de estudios; y el "Benjamín", José, terminando también sus estudios de pregrado en psicología.

Desde sus inicios como staff en la UC y junto a nuestro maestro común, el Dr. Jorge Urzúa, Guillermo se dedicó tiempo completo a la anestesia cardiovascular. A poco andar, se empieza a planificar un proyecto de desarrollo de cirugía de cardiopatías congénitas en lactantes y recién nacidos. Es así, que junto a Janet y su hijo Nicolás, parte el año 87 a realizar un Fellow en Anestesia Cardiotorácica en el Harefield Hospital de Londres, Inglaterra, lugar donde nacería su hijo Tomás; y donde participa activamente en el equipo del destacado cirujano cardiotorácico Sir Magdi Yacoub, precursor de múltiples técnicas y avances en el tratamiento quirúrgico de cardiopatías congénitas y trasplante cardiaco.

Vuelve un par de años después a la UC, dándole un gran impulso a esta área quirúrgica, que desde entonces ha crecido significativamente, transformándose en uno de los pilares de nuestro quehacer clínico, docente y académico. Mientras tanto, la familia se completa con la llegada de su hijos Constanza y José.

En una editorial de 2010 de la Revista Chilena de Anestesia, el Dr. Ricardo Bustamante nos informaba que un comité de sabios reunido por el directorio de la Sociedad de Anestesia de la época había elaborado un protocolo estandarizado para la designación de los Maestros de la Anestesiología. Entre los requisitos se dispone que éstos deben ser mayores de 65 años, haber permanecido en la Sociedad durante 25 o más años y haber realizado un trabajo gremial o científico por al menos 10 años, ya sea como miembro del directorio, miembro del comité editorial de la Revista de Anestesiología de Chile, miembro de comités de subespecialidades, participación activa en congresos (organización, conferencias, mesas redondas o presentación de temas libres) o participación en otras actividades (gremiales, elaboración de recomendaciones, etc.). Además, "Ios Maestros de la Anestesiología deben tener publicaciones, sin especificar tipo, lugar o relevancia de la publicación, que pueden ser temas subjetivos de evaluar. Concomitantemente, debe existir consenso en el Directorio de la Sociedad en que el candidato ha participado en forma decisiva en la formación de anestesiólogos y en el desarrollo de la especialidad. Este requisito debe ser aprobado con el voto de al menos 6 de los 7 miembros (quórum calificado)" (Rev Chil Anest 2010, Vol 39 (4), 251-253).

Al mirar el extenso currículo que ha construido el Dr. Lema durante su vida profesional y académica es fácil darse cuenta de que cumple con absolutamente todos los requisitos necesarios para obtener este reconocimiento. No amerita extenderme en este punto, a riesgo de aburrir más de lo necesario, pero solo quisiera destacar algunos hitos que nos hablan de la integralidad de su quehacer académico y docente, y de lo rápida (por no decir meteórica) que fue su carrera.

- A nivel de carrera académica, ingresa a la UC como instructor asociado a los 28 años, a los 29 es nombrado instructor categoría ordinaria, a los 31 profesor auxiliar, a los 38 profesor adjunto y a los 52 ya era profesor titular.

- En cuanto a su labor gremial y de extensión, el año 89 es nombrado vicepresidente de la Sociedad de Anestesiología de Chile y conjuntamente presidente de la FASA (Federación de Asociaciones Sudamericanas de Anestesiología), ocupando luego la presidencia de nuestra Sociedad de los 91 al 93. Después de su periodo como past-president ha seguido ligado a ella en diversas tareas (Comité de Investigación, Comité Científico, Comité de Anestesia Cardiovascular, etc.).

- Miembro de Conacem y Asofamech desde el año 93, ocupa la presidencia de la Comisión de Anestesiología de esta última. Par evaluador Ápice desde el 2010.

- Destacada labor como editor de la Revista de Anestesiología de Chile por 14 años. Miembro del comité editorial de diversas revistas científicas a nivel nacional e internacional, destacando la edición en español de Anestesia \& Analgesia, el Journal of Cardiothoracic and Vascular Anesthesia, el World Journal of Nephrology y la revista Perfusion.

- Dentro de las tareas que le ha encomendado la Facultad de Medicina de la UC ha ocupado, entre otros, un cargo en la Comisión de Graduados por 10 años y en el Consejo de Facultad por 4 años; es actualmente miembro de la Comisión de Carrera y Evaluación Académica de las Carreras de la Salud, además de director del Programa de Farmacología y Toxicología desde su reciente creación en 2017.

- Los aportes de Guillermo en investigación y publicaciones son muy numerosos: más de 130 presentaciones en cursos y congresos; sobre 120 publicaciones ISI, 51 de ellas como primer autor; 10 proyectos de investigación UC y 5 proyectos Fondecyt, 2 de ellos como investigador principal, ambos en el área de la función renal durante circulación extracorpórea, tema en el cual es una autoridad reconocida a nivel internacional.

- En las dos últimas ediciones del Miller, Guillermo ha aparecido como coautor de un capítulo sobre la visión de la práctica de la anestesia en el mundo.

- Finalmente, y probablemente en el rol más importante a considerar para ser postulado a este reconocimiento - en relación a su labor docente y formativa de las nuevas generaciones, somos casi 400 los residentes y ahora anestesiólogos que hemos disfrutado y "sufrido", entre dobladitas con palta y tortas de hojarasca, el trabajo docente de Guillermo, obviamente en conjunto con el del resto de la División de Anestesia, que en estos 40 años ha crecido enormemente, hasta llegar a conformarla actualmente más de 40 especialistas, 9 o 10 de los cuales somos anestesiólogos cardiovasculares.

- Guillermo ha sido el líder natural del grupo de anestesiólogos cardiovasculares de la UC por más de 30 años, y uno de los más distinguidos y respetados en este rubro a nivel nacional y regional, siempre trabajando y luchando porque la subespecialidad fuera reconocida como tal. Fue el impulsor y jefe durante 14 años del primer y único programa de formación reconocido en Anestesia Cardiovascular del país. Desde que la Dra. Carla Sacco fuera nuestra primera becada oficial de Anestesia Cardiovascular, el año 1990, 26 subespecialistas han recibido este título y están trabajando desde Punta Arenas hasta Antofagasta, pasando por Temuco, Concepción, Talca y diversos hospitales públicos y privados de Santiago, así como en Bolivia y Ecuador. Otro importante grupo de colegas ha realizado estadías de perfeccionamiento de duración variable (incluyendo anestesiólogos de distintos países de Centro y Sudamérica). También Guillermo ha sido 
un importante defensor e impulsor, siguiendo las enseñanzas de Jorge Urzúa, de que la perfusión o circulación extracorpórea continúe estando bajo la supervisión y la enseñanza de los anestesiólogos. Es así como llevamos más de 30 años formando perfusionistas en un programa de 1 año de duración, profesionales que trabajan en todo Chile y en varios países de Latinoamérica. Desde el año 2020 este programa es reconocido por nuestra Universidad como un Post Título para profesionales de la salud, a cargo de la División de Anestesia. Por otra parte, el Dr. Lema, gracias a sus contactos internacionales, ha guiado y ayudado a decenas de residentes a conseguir rotaciones y estadías de perfeccionamiento en distintos lugares del mundo y creó y dirigió, junto a otros destacados colegas y amigos, el Comité de Anestesia Cardiovascular de la Sociedad de Anestesia.

Al revisar su extenso trabajo, uno se pregunta cómo se puede hacer tanto y en tantos ámbitos diferentes. Personalmente me tocó conocer y trabajar directa o indirectamente con 8 o 9 maestros de la anestesiología chilena (UC, directorio de la Sociedad, congresos, revista, etc.). En ellos encontré personalidades muy diferentes, con matices, formas y talentos diversos, pero a la vez con características similares, iguales en voluntad, actitud, trabajo y generosidad, y en la necesidad de traspasar a otros lo aprendido, de multiplicar, de sembrar, de dar. Todos conocemos colegas que lo hacen muy bien, muy dotados, que uno no duda en recomendar cuando te piden una opinión, pueden hacer cosas extraordinarias, pero se quedan ahí, lo hacen cuando es necesario y luego el talento se guarda, se oculta para que otros no lo vean, para que otros no aprendan, no hacen escuela, no forman discípulos que puedan hacerlo igual o mejor que ellos, están centrados en sí mismos y son egoístas con sus talentos. Guillermo, afortunadamente, no pertenece a este grupo.

Para terminar uso como analogía, guardando las proporciones, lo que nos decía un amigo religioso con respecto a la definición de "santidad": "un santo no es alguien anormal que hace cosas extraordinarias; un santo es alguien muy normal, que hace cosas normales extraordinariamente bien". Esta definición se le acerca muy bien a lo que creo que ha significado el Dr. Guillermo Lema para la anestesiología chilena: no es un sabio ni un genio, no es un iluminado ni un superdotado, es alguien muy normal, inteligente, muy trabajador y estudioso, muy voluntarioso y talentoso, que además de poner todo este talento al servicio de sus pacientes, principal labor de nuestro quehacer médico, ha puesto todo su ser en trascender su conocimiento y experiencia a sus discípulos, en hacer crecer, en multiplicar... un imprescindible.

Por todo ello, creo que el Directorio de la Sociedad de Anestesiología de Chile ha hecho lo correcto en distinguirlo entre sus pares y es para mí una tremenda alegría y un gran orgullo darle la bienvenida al Dr. Guillermo Lema Fuxman como el décimo sexto Maestro de la Anestesiología de Chile. 